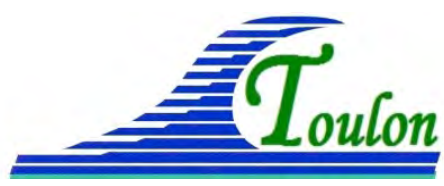

XIV èmes Journées Nationales Génie Côtier - Génie Civil Toulon, 29 juin au $1^{\text {er }}$ juillet 2016

DOI:10.5150/jngcgc.2016.072 (C) Editions Paralia CFL

disponible en ligne - http://www.paralia.fr - available online

\title{
Modélisation du risque de submersion marine intégrant les défaillances d'ouvrages. Application à l’île de Ré.
}

\author{
Jérémy DUGOR ${ }^{1}$, Didier RIHOUEY ${ }^{1}$, Jentsje VAN DER MEER ${ }^{2}$
}

1. CASAGEC INGENIERIE, 18 rue Maryse Bastié, Zone de Maignon, 64600 Anglet, France. dugor@casagec.fr, rihouey@casagec.fr

2. VAN DER MEER CONSULTING B.V, Ljouwerterdyk 55 A, 8491 ML Akkrum, the Netherlands jm@vandermeerconsulting.nl

\section{Résumé :}

La tempête Xynthia, qui a touché les côtes atlantiques françaises dans la nuit du 27 au 28 février 2010, a été à l'origine de phénomènes de submersion et d'érosion d'une rare intensité, notamment sur les côtes vendéennes et en Charente-Maritime, causant la mort de 59 personnes et près de deux milliards d'euros de dommages. Le passage de la tempête au maximum de son creusement coïncidait avec la pleine mer d'une marée de vive-eau de coefficient 102 et des fortes houles comprises entre 6 et $7 \mathrm{~m}$, provoquant une surcote de l'ordre de 1,50 m à La Rochelle. Sur certains territoires comme l'Ile de Ré, les défaillances des digues sont responsables d'une part importante de l'ampleur de la submersion. Au-delà du mauvais état avéré de nombreux ouvrages, le retour d'expérience a montré que les surverses et les franchissements par paquets de mer étaient en majorité responsables des défaillances des digues.

La représentation réaliste des défaillances d'ouvrages constitue donc un enjeu majeur pour évaluer le risque de submersion sur les secteurs protégés par des digues. Cet article présente un système de modélisation numérique couplé (Télémac2D/Tomawac), permettant de reproduire de façon précise la submersion occasionnée par la tempête Xynthia sur l'Ile de Ré. Le modèle mis en place consiste en l'élaboration d'un maillage régional unique capable de représenter au mieux les processus de génération de la surcote (marée, pression atmosphérique, vent, houle et setup) et d'éviter la surestimation des niveaux d'eau maximaux en autorisant les débordements au niveau des ouvrages de protection (BERTIN et al., 2014a \& 2014b). Un module spécifique a également été développé pour intégrer les franchissements par paquets de mer calculés sur chaque ouvrage.

Ce modèle a été utilisé pour calculer les sollicitations hydrauliques (surverse, impact des vagues, franchissements par paquets de mer) sur l'ensemble des digues de l'île de Ré au cours de la tempête. De plus, une inspection des ouvrages et un diagnostic de leur fonctionnement face à l'évènement Xynthia ont conduit à définir des règles de défaillances (moment de rupture, vitesse de formation, largeur et hauteur finale) en 


\section{Thème 7 - Risques côtiers}

accord avec l'état de l'art. L'application généralisée de ces règles de défaillances à l'ensemble du système de protection de l'Ile permet de représenter précisément l'emprise de submersion observée dans le Retour d'Expérience Xynthia (REX, CDC ILE DE RÉ, 2014).

Cette méthode, appliquée à d'autres évènements que Xynthia (intensité et direction différentes), pour des ouvrages de protection modifiés ou avec une prise en compte du réchauffement climatique, permet d'appréhender le risque de submersion marine sur l’Ile de Ré en intégrant le système de protection et ses défaillances.

Mots-clés : Risques côtiers, Brèches, Ouvrages de protection, Franchissements par paquets de mer, Surverse, Xynthia, Ile de Ré, Retour d'expérience, Modélisation numérique.

\section{Introduction}

La tempête Xynthia a été à l'origine de phénomènes de submersion et d'érosion d'une rare intensité, notamment sur les côtes de Charente Maritime, où une surcote de l'ordre de 1,50 m à La Rochelle a été enregistrée. Sur l'île de Ré, les défaillances des digues (érosion de crêtes, brèches) ont été une cause importante de l'ampleur de la submersion. Au-delà du mauvais état avéré de nombreuses digues, le Retour d'Expérience Xynthia (REX) a montré que les surverses et les franchissements par paquets de mer étaient en majorité responsables des défaillances d'ouvrages. Au total, ce sont 2400 ha qui ont été submergés (1/3 de l'île) et 5,9 km de défaillances sur un linéaire de 65,5 km de digues. La figure 1 permet de situer les submersions et les défaillances. A la vue de ces chiffres, il apparait donc nécessaire de prendre en compte les défaillances d'ouvrages pour analyser l'aléa submersion marine sur ce territoire.

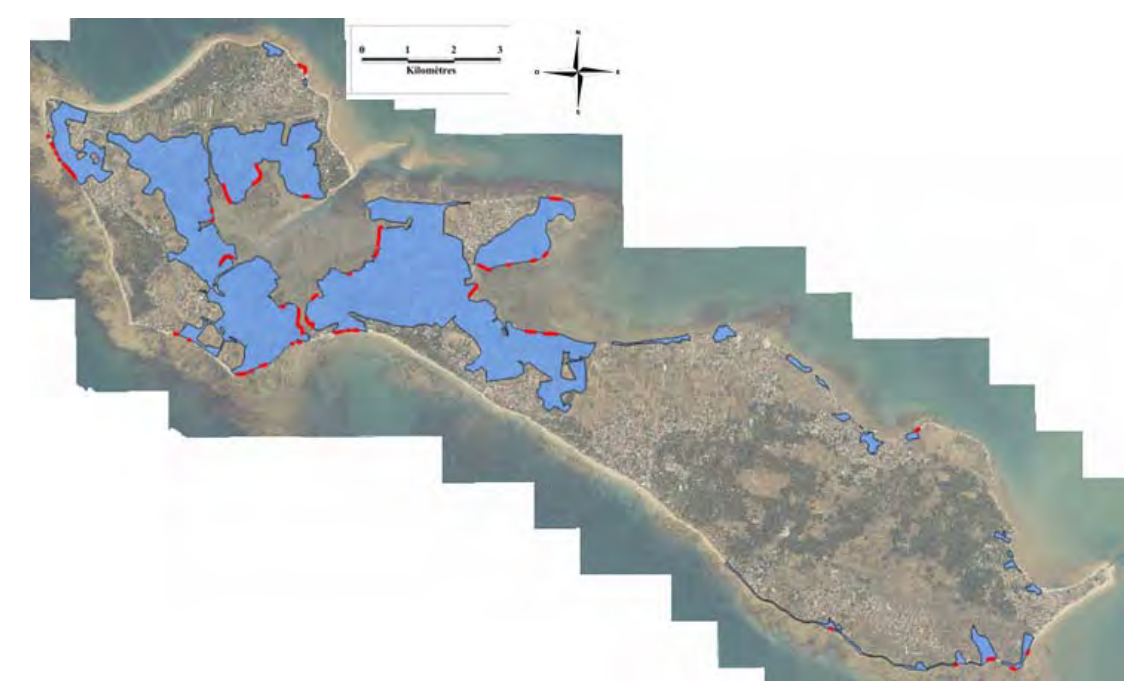

Figure 1. Emprise de submersion (bleue) et localisation des défaillances (lignes rouges) suite à la tempête Xynthia (Source : retour d'expérience REX, CDC ILE DE RÉ, 2014). 


\section{XIV ${ }^{\text {èmes }}$ Journées Nationales Génie Côtier - Génie Civil \\ Toulon, 29 juin au $1^{\text {er }}$ juillet 2016}

\section{Description du modèle mis en œuvre}

\subsection{Emprise, maillage et forçages}

Le modèle (couplage total Telemac/Tomawac) consiste en un maillage régional unique (figure 2) qui permet de représenter au mieux les processus de génération de la surcote (marée, pression atmosphérique, vent, houle et setup induit par les vagues) et intègre la partie terrestre, ce qui évite la surestimation des niveaux d'eau maximaux en permettant les débordements au niveau des ouvrages (BERTIN et al., 2014a \& 2014b).
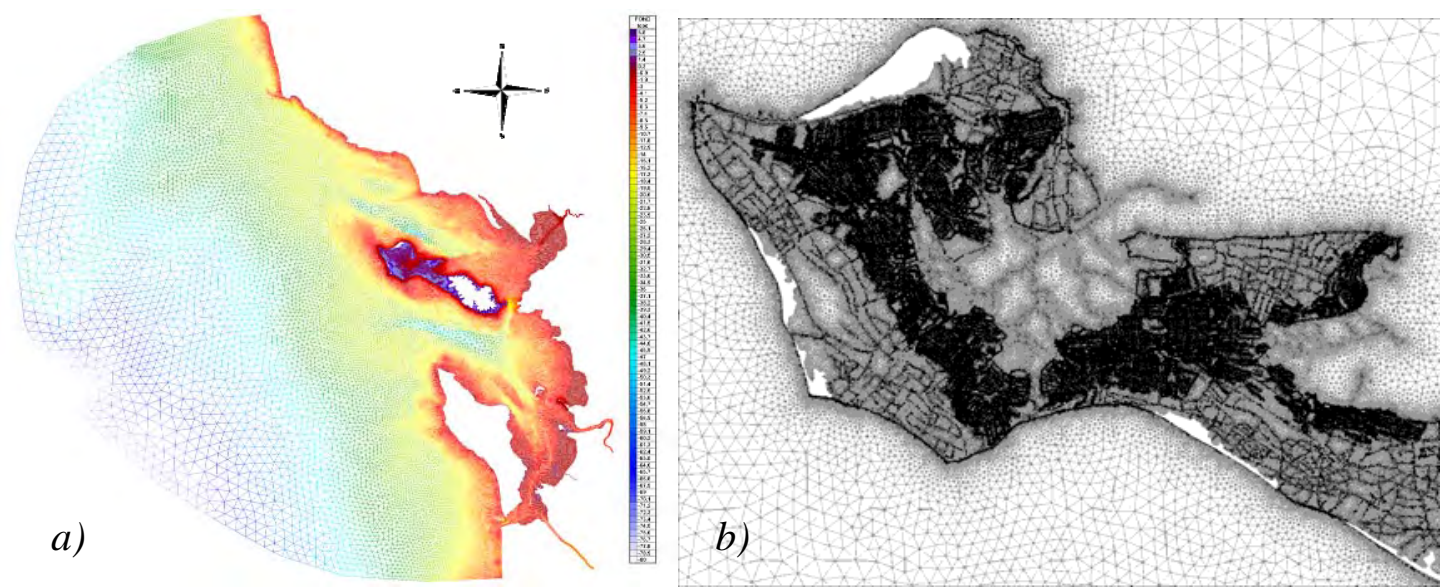

Figure 2. a) Emprise du modèle régional. b) Détail du maillage sur le canton Nord.

La topographie est issue d'un levé Lidar de 2011 et des levés topographiques supplémentaires ont été réalisés afin d'avoir une représentation précise de l'ensemble des ouvrages dans leurs configurations actuelles. Les caractéristiques des digues qui ont cédé durant Xynthia ont été prises en compte dans le calage du modèle. Le modèle complet est composé de 369464 nœuds de calcul. Pour la partie maritime, les tailles des mailles sont comprises entre $20 \mathrm{~m}$ à la côte jusqu'à $3 \mathrm{~km}$ au large. Pour la partie terrestre, les tailles des mailles sont comprises entre 1 et $50 \mathrm{~m}$. Les marais et les canaux d'irrigations sont représentés distinctement dans le maillage, permettant d'assurer les liaisons hydrauliques. Les ouvrages de type écluse, buse et clapet sont intégrés dans la modélisation. La nature des terrains et les frottements qu'ils représentent sont pris en compte avec une loi de Strickler. Le découpage des coefficients a été réalisé avec la base de données Corine Land Cover et des documents d'occupation du sol fournis par les services techniques locaux. La valeur des coefficients a ensuite été ajustée pour caler le modèle à terre. Ainsi, si les bâtiments ne sont pas intégrés dans le maillage, leur présence est partiellement compensée par une modification locale de la rugosité.

Les forçages suivants ont été imposés au modèle : la marée astronomique est forcée sur la frontière océanique grâce au modèle de marée TPXO, le vent et la pression atmosphérique sont issus du modèle AROME (Météo France). Ils sont intégrés de 


\section{Thème 7 - Risques côtiers}

manière variable dans l'espace et dans le temps et permettent de calculer la surcote atmosphérique. L'effet du gradient de pression atmosphérique sur le niveau d'eau est intégré par la méthode du baromètre inversé. Les vagues sont forcées à la frontière océanique du modèle par des valeurs qui permettent de bien représenter les observations du houlographe présent au large de l'île d'Oléron. Le couplage total Télémac2D/Tomawac permet de calculer les courants de circulation sur le littoral et également d'évaluer le setup induit par les vagues.

Enfin, le module de brèche intégré dans le code Télémac permet de représenter fidèlement les défaillances (vitesses, largeur, moment d'initialisation et hauteur finale).

\subsection{Intégration des franchissements par paquets de mer}

Un module spécifique a été développé dans TELEMAC pour intégrer les franchissements par paquets de mer (DUGOR et al., 2014). Les différents paramètres suivants sont récupérés à chaque pas de temps de la simulation :

a) niveau d'eau $\left(Z_{\text {eau }}\right)$ au pied de l'ouvrage,

b) hauteur $\left(H_{m 0}\right)$, direction $(\beta)$ et période des vagues $\left(T_{m-1,0}\right)$ à une distance connue du pied de l'ouvrage à l'extérieur de la zone de déferlement,

c) hauteur de la crête $\left(Z_{c}\right)$, la revanche de crête $\left(R_{c}\right)$ et pente de l'ouvrage $(\alpha)$.

Le débit " $q$ " franchissant les ouvrages de protection est alors calculé par les formules décrites dans le manuel EUROTOP (2007) et le guide Enrochement (CIRIA/CUR/CETMEF, 2009) :

Si $\gamma_{b} \cdot \xi<2$ alors $\frac{q}{\sqrt{g \cdot H_{m 0}^{3}}}=\frac{0,067}{\sqrt{\tan (\alpha)}} \cdot \gamma_{b} \cdot \xi \cdot \exp \left(-4,3 \cdot \frac{R_{c}}{H_{m 0}} \cdot \frac{1}{\gamma_{b} \cdot \gamma f \cdot \gamma \beta \cdot \xi}\right)$

Si $\gamma_{b} \cdot \xi>2$ alors $\frac{q}{\sqrt{g \cdot H_{m 0}^{3}}}=0,2 \cdot \exp \left(-2,3 \cdot \frac{R_{c}}{H_{m 0}} \cdot \frac{1}{\gamma \cdot \cdot \gamma \beta}\right)$

Avec : $S_{0}=\frac{2 \cdot \pi \cdot H_{m 0}}{g \cdot T_{m-1,0}^{2}}$ la cambrure des vagues et $\xi=\frac{\tan (\alpha)}{\sqrt{S_{0}}}$ le paramètre de déferlement.

Les coefficients $\gamma_{b}, \gamma f, \gamma \beta$ représentant respectivement les facteurs d'amortissements correspondant à la présence d'éventuelles bermes, la rugosité des revêtements des ouvrages et l'influence de l'angle d'incidence des vagues. Les valeurs de ces paramètres sont définies selon les recommandations du manuel EUROTOP (2007). Lorsque la revanche de crête $\left(R_{c}\right)$ est nulle ou négative, des formules adaptées du manuel EUROTOP (2007) sont utilisées. Le débit q est ensuite imposé à l'arrière de chacune des structures soumises aux franchissements par paquets de mer. En raison des phénomènes irréguliers dans la zone de déferlement, les résultats du modèle de propagation de houle ont été extraits à quelques dizaines de mètres du pied de l'ouvrage, en fonction de la configuration de la bathymétrie. Les données sont ensuite ramenées au pied de l'ouvrage (cf. figure 3) avec les formules décrites dans le manuel EUROTOP (2007). Ces formules dépendent du paramètre de déferlement ( $\xi$ ), de la 


\section{XIV èmes Journées Nationales Génie Côtier - Génie Civil \\ Toulon, 29 juin au $1^{\text {er }}$ juillet 2016}

cambrure des vagues $\left(S_{0}\right)$, de la pente de plage et de la profondeur d'eau au pied de l’ouvrage $(h)$. Cette chaîne de calcul est exécutée par le modèle à chaque pas de temps.
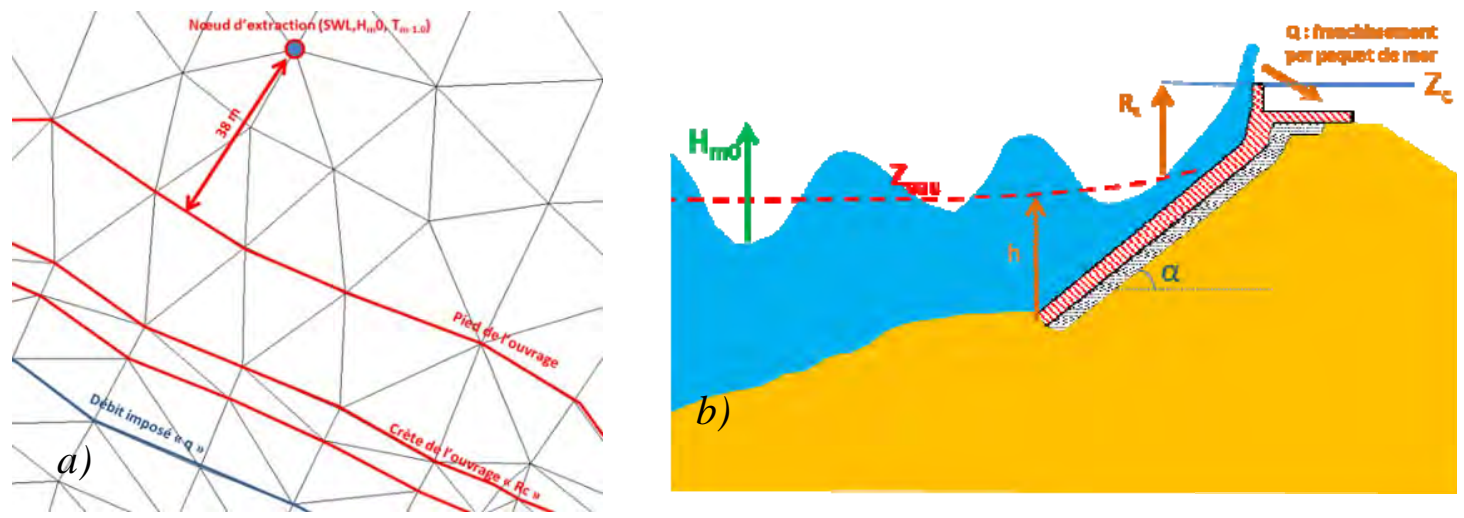

Figure 3. a) Exemple de configuration d'ouvrage dans le maillage du modèle numérique pour le calcul de franchissement par paquets de mer " $q$ " et b) définition des principales variables utilisées pour les calculs.

\subsection{Calage du modèle à la côte}

Le modèle représente bien le niveau d'eau sur l'ensemble des trois marégraphes les plus proches de la zone d'étude (les Sables d’Olonne, la Pallice (cf. figure 4a) et la Cotinière) avec un biais inférieur à $10 \mathrm{~cm}$ sur le cycle de marée complet. Afin de s'assurer de la bonne représentation du gradient de niveau d'eau le long de l'île de Ré, des laisses de submersion sur le littoral ont été levées au niveau des ports de SaintMartin-de-Ré et d'Ars-en-Ré, où l'effet des vagues est négligeable (cf. figure 4b). La comparaison de ces observations avec les résultats du modèle montre que la hauteur d’eau est bien représentée avec une différence inférieure à 5 cm.
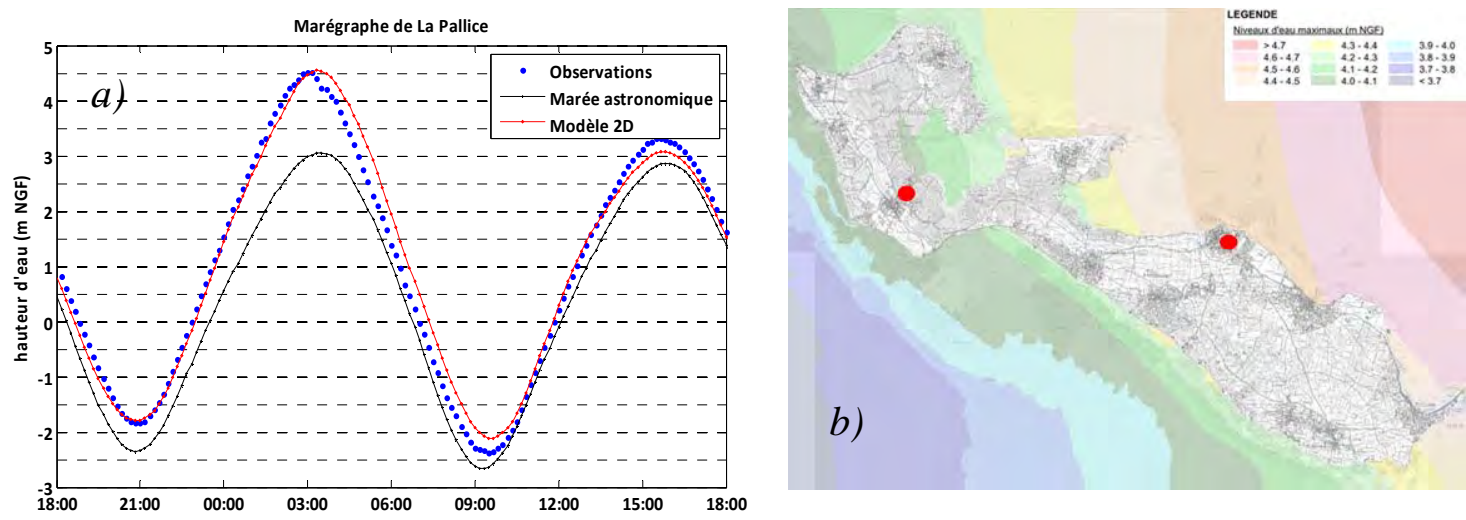

Figure 4. a) Comparaison des niveaux d'eau modélisés et mesurés au marégraphe de la

Pallice et b) carte des niveaux d'eau maximaux avec la localisation des laisses de submersion qui ont permis de caractériser les cotes d'eau sur le littoral de l'île de Ré 


\section{Analyse des brèches et attribution de règles de défaillance}

\subsection{Généralités}

Les facteurs déclenchant les défaillances dépendent de la structure de la digue et des conditions aux limites hydrauliques et morphologiques. On distingue 4 grands facteurs hydrauliques (cf. figure 5a) initiant des défaillances structurelles : les franchissements par paquets de mer, la surverse, l'impact des vagues et les infiltrations. La figure $5 \mathrm{~b}$ illustre un hydrogramme "type" d'une brèche en formation à travers une digue. Les mécanismes de défaillance peuvent être représentés par quatre phases distinctes: l'initialisation, la transition, la formation, le développement.
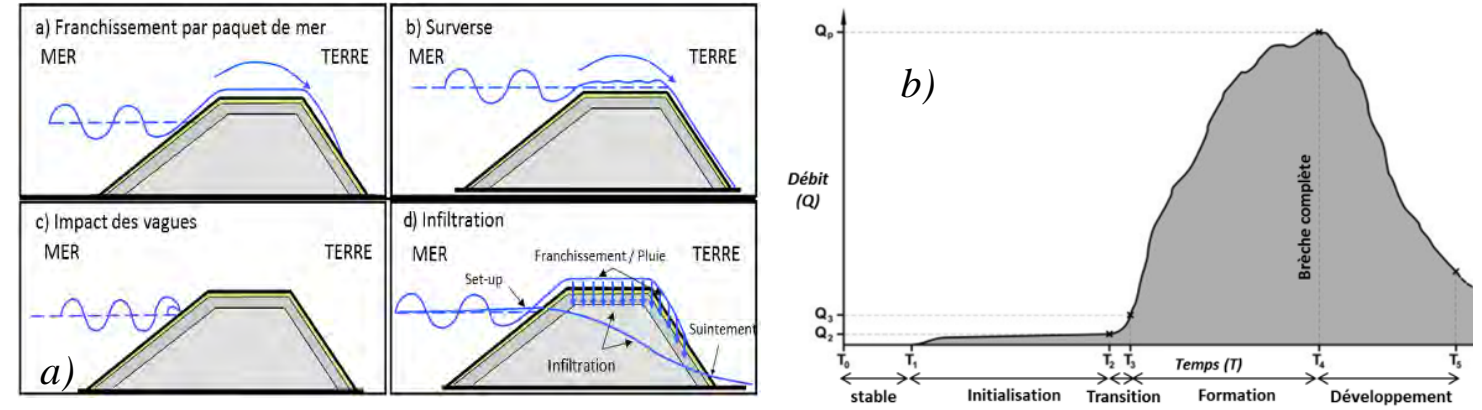

Figure 5. a) Facteurs hydrauliques initiant les défaillances structurelles.

b) Hydrogramme "type" de formation d'une brèche (d'après MORRIS et al., 2008).

En pratique, la forme et la durée de l'hydrogramme d'une brèche sont déterminées par la charge hydraulique (volume d'eau retenu par la digue), la variation du niveau d'eau (marée), l'état de la digue... Enfin, la cinétique d'une défaillance peut changer significativement en fonction du type de matériau utilisé dans le corps de digue (cf. figure 6) :

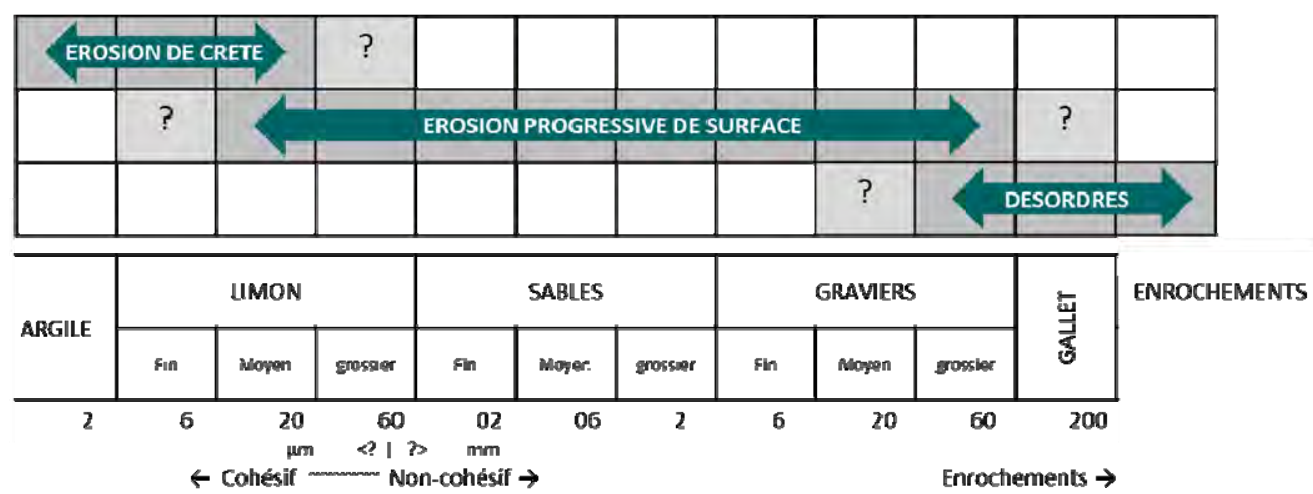

Figure 6. Classification du comportement des brèches en fonction du type de matériau (d'après MORRIS et al., 2008). 


\section{XIV èmes Journées Nationales Génie Côtier - Génie Civil \\ Toulon, 29 juin au $1^{\text {er }}$ juillet 2016}

\subsection{Analyse des brèches}

Le Retour d'Expérience Xynthia (REX) renseigne sur la largeur et la hauteur finale des brèches. Cependant, le moment d'initialisation et la durée de formation ne sont pas connus. Grâce au modèle numérique et aux calculs de franchissements par paquets de mer, ces paramètres ont pu être évalués pour chaque défaillance en fonction de la typologie de la digue, des sollicitations hydrauliques et de la configuration finale de la brèche. Un exemple d'analyse de rupture est donné ci-dessous pour deux digues (D1 et D2). Ces digues sont composées principalement de bri (une argile locale très cohésive) dont les faces internes ne sont pas protégées et les crêtes sont à une altitude de $4 \mathrm{~m}$ NGF. D1 est localisée sur un secteur abrité des vagues $\left(H_{s \max }<0,2 \mathrm{~m}\right)$ et D2 est exposée aux clapots d'une baie semi-ouverte $\left(H_{s}\right.$ max $\left.\sim 1 \mathrm{~m}\right)$. Les observations de terrain indiquent les éléments suivants :

a) Pour D1, l'initialisation est causée par une surverse. Le moment d'initialisation et l'arrêt de la formation de la brèche sont donc déterminés avec le niveau d'eau (cf. figure 7a, courbe noire),

b) Pour D2, le facteur déclenchant l'initialisation est le franchissement par paquets de mer généré par le clapot, qui entraîne une érosion régressive de la face interne. Grâce au modèle numérique, les volumes de franchissements ont pu être évalués en fonction de la marée et des vagues, ce qui a permis de déterminer l'initialisation de la brèche (cf. figure $7 \mathrm{a}$, courbe rouge).

Pour les deux digues, la connaissance de la hauteur finale de la brèche par le REX et l'analyse de l'initialisation a permis de déduire les vitesses de formation $(0,5 \mathrm{~m} / \mathrm{h})$.

Un chronogramme décrit ces deux exemples sur la figure $7 \mathrm{a}$ et un cliché d'une formation de brèche sur une digue en argile non exposée aux vagues est présenté sur la figure $7 \mathrm{~b}$.
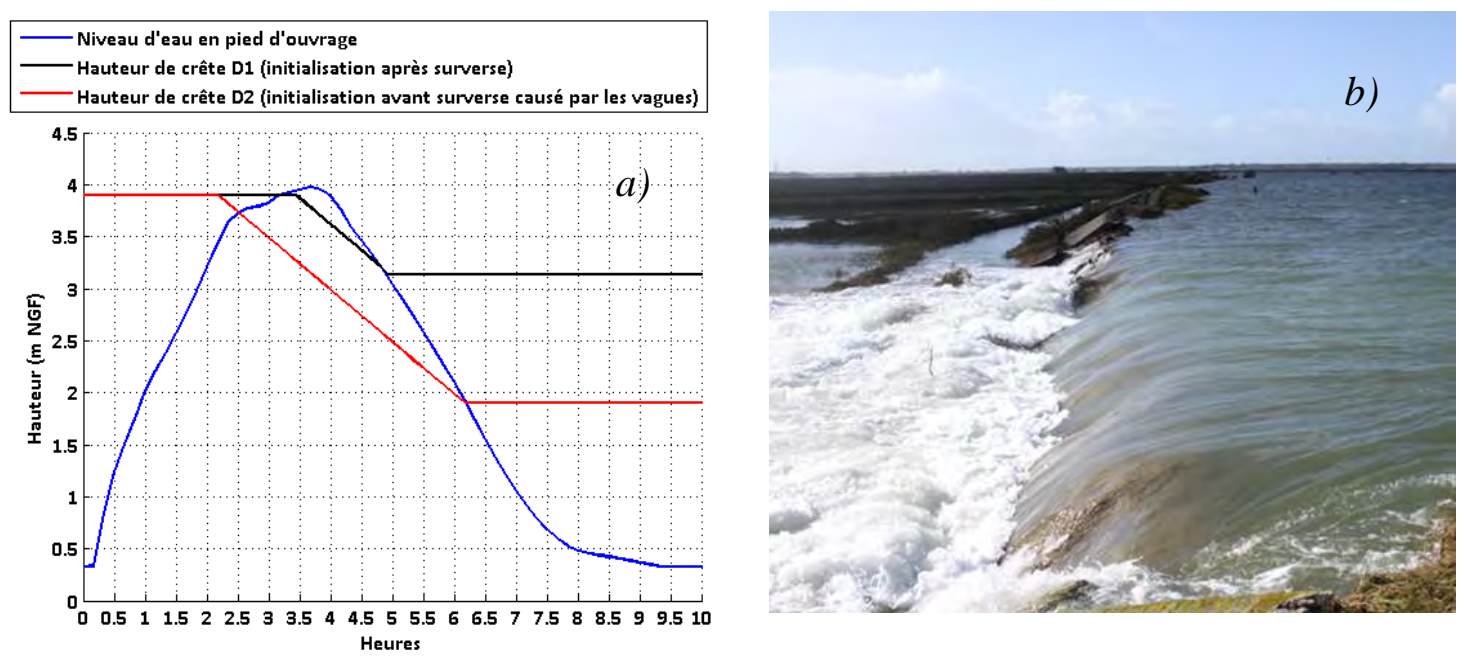

Figure 7. a) Chronogramme décrivant la formation de 2 brèches pour 2 digues similaires et b) cliché d'une surverse sur une digue en bri (source : LPO) 


\section{Thème 7 - Risques côtiers}

\subsection{Attribution des règles de défaillance}

En accord avec l'état de l'art et le REX, les vitesses de formation des défaillances ont été définies sur la base des 3 grands types de matériaux constituant les corps des digues : sable $(10 \mathrm{~m} / \mathrm{h})$, calcaire compacté $(1 \mathrm{~m} / \mathrm{h})$ et argile $(0.5 \mathrm{~m} / \mathrm{h})$.

Ensuite, l'analyse des brèches a permis de classer les processus des défaillances similaires et de dresser des règles communes pour chacun de ces processus. Afin de faciliter l'analyse des modes de rupture des digues durant la tempête Xynthia, les règles concernant les ouvrages non-exposés aux vagues ont été dissociées. Au total 18 règles ont été définies. Un exemple de quelques règles est donné dans le tableau 1.

Tableau 1. Exemple de règles associées aux secteurs exposés aux vagues.

\begin{tabular}{|c|c|c|c|c|c|}
\hline ID & Type de Structure & $H_{s}(m)$ & $Q(L / s / m)$ & Longueur (\%) & $\begin{array}{l}\text { Altitude } \\
\text { Finale }\end{array}$ \\
\hline I.1 & Digue en maçonnerie surmontée d'un parapet & $>1,5$ & & $50 \%$ du secteur & $\begin{array}{l}Z_{\text {Crête }}- \\
H_{\text {Parapet }}\end{array}$ \\
\hline$I .2$ & Digue en maçonnerie sans protection arrière & & $>30$ & $40 \%$ du secteur & $4 \mathrm{~m} \mathrm{NGF}$ \\
\hline$I .3$ & Cordon de galet sans protection & $>0,5$ & $>5$ & $50 \%$ du secteur & $Z_{\text {Crête }}-1 m$ \\
\hline I.4 & Enrochements (1-5 T) & & $>100$ & $50 \%$ du secteur & $\begin{array}{l}\text { Terrain } \\
\text { naturel }\end{array}$ \\
\hline$I .5$ & Gabions & & $>30$ & Tout le linéaire & $\begin{array}{l}\text { Terrain } \\
\text { naturel }\end{array}$ \\
\hline$\ldots$ & $\ldots$ & $\cdots$ & $\cdots$ & $\cdots$ & $\cdots$ \\
\hline
\end{tabular}

Ces règles ont ensuite été appliquées dans le modèle, à l'ensemble des digues de l'île de Ré dans leurs configurations de 2010 avec les sollicitations hydrauliques de la tempête Xynthia.

\subsection{Validation de la méthode}

La comparaison des résultats du modèle aux observations du REX (niveau d'eau et emprise de submersion) donne de bons résultats avec une tendance générale à la surestimation des hauteurs d'eau $(-0,07 \mathrm{~m}<\Delta h<0,28 \mathrm{~m}$ sur les laisses de crue). Concernant l'emprise de submersion, elle est relativement bien représentée avec également une légère surestimation par rapport au REX. Ces résultats montrent que les règles de défaillances et les hypothèses liées à la cinétique et à l'initialisation sont cohérentes avec la réalité. La surestimation est expliquée par l'application des règles de défaillance à l'ensemble des digues de l'Ile de Ré, ce qui implique logiquement un linéaire de brèches plus important que le REX.

L'application de cette méthode sur d'autres territoires et d'autres tempêtes permettrait de renforcer la validation de ces règles de défaillance. 


\section{XIV èmes Journées Nationales Génie Côtier - Génie Civil \\ Toulon, 29 juin au $1^{\text {er }}$ juillet 2016}

\section{Conclusions}

La méthode mise en place permet d'évaluer le risque de submersion marine en intégrant la défaillance des ouvrages de manière réaliste. Elle est basée sur un outil de modélisation (Télémac/Tomawac) intégrant un module de calcul des franchissements par paquets de mer (DUGOR et al., 2014) et un module de génération de brèches.

L'analyse fine du système de protection et du retour d'expérience de la tempête Xynthia sur les ouvrages de l'Ile de Ré a permis de définir des règles de défaillances en fonction des sollicitations hydrauliques et de la typologie des ouvrages.

L'application de ces règles à l'ensemble des digues de l'Ile de Ré dans leurs configurations de 2010 avec les sollicitations hydrauliques de la tempête Xynthia donne des résultats satisfaisants avec une tendance à la surestimation par rapport au REX, ce qui va dans le sens de la sécurité.

Ce modèle et ces règles peuvent être appliqués à d’autres types de tempêtes (direction, intensité, durée...) et intégrer l'élévation du niveau de la mer causé par le réchauffement climatique. Ces modifications font varier les sollicitations hydrauliques et par conséquent le scénario de défaillance des ouvrages. In fine, la mise en œuvre d'une étude de sensibilité du scénario de défaillance permet de caractériser l'aléa submersion de façon précise et exhaustive.

\section{Références bibliographiques}

BERTIN X., LI K., ROLAND A., ZHANG Y.J., BREILH J.F, CHAUMILLON E. (2014a). Simulation numérique de la submersion marine associée à la à la tempête Xynthia, février 2010. XIII ${ }^{\text {èmes }}$ Journées Nationales Génie Côtier - Génie Civil ; Dunkerque, 2-4 juillet 2014, pp 879-888. http://dx.doi.org/10.5150/jngcgc.2014.097

BERTIN X., LI K., ROLAND A., ZHANG Y.J., BREILH J.F, CHAUMILLON E. (2014b). A modeling-based analysis of the flooding associated with Xynthia, central Bay of Biscay. Coastal Engineering, Vol. 94, pp 80-89. http://dx.doi.org/10.1016/j.coastaleng.2014.08.013

CIRIA/CUR/CETMEF (2009). Guide Enrochement. Utilisation des enrochements dans les ouvrages hydrauliques. Traduction française du Rock Manual, PM09.01, CETMEF. DUGOR J., RIHOUEY D., LANG P. (2014). Flood modelling on Ile de Ré during Xynthia. $21^{\text {st }}$ Telemac Mascaret User Conference; Grenoble, $15-17^{\text {th }}$ October.

CDC ILE DE RÉ -Communauté de Communes- (2014). Retour d'expérience Xynthia (REX) - Défaillance des ouvrages de défense contre la mer.

EUROTOP (2007). Wave overtopping of sea defences and related structure: Assessment manual. Co-authors: Pullen, T., Allsop, W., Bruce, T., Kortenhaus, A., Schütrumpf, H \& Van Der Meer, J.W.

MORRIS M., HANSON G., HASSAN M. (2008). Improving the accuracy of breach modelling: why are we not progressing faster? Journal of Flood Risk Management, Vol. 1(4), pp 150-161. http://dx.doi.org/10.1111/j.1753-318X.2008.00017.x 
Thème 7 - Risques côtiers 